\title{
Infant mortality across species. A global probe of congenital abnormalities
}

\author{
Alex Bois ${ }^{1}$, Eduardo M. Garcia-Roger ${ }^{2}$, Elim Hong $^{3}$, Stefan Hutzler ${ }^{4}$, Ali Irannezhad ${ }^{5}$

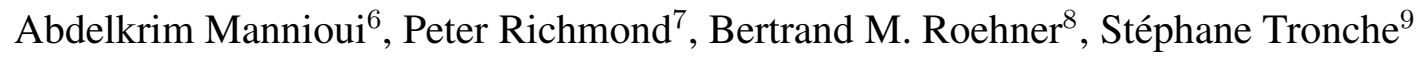

Version of 2 May 2019

Key-words: Congenital anomalies, malformations, infant mortality, manufacturing defects

1: Aquatic facility, Pierre and Marie Curie Campus, Sorbonne University, Paris, France.

Email: alex.bois@upmc.fr

2: Institut Cavanilles de Biodiversitat I Biologia Evolutiva, University of València, Spain.

Email: eduardo.garcia@uv.es

3: Neuroscience Laboratory, Sorbonne University and INSERM (National Institute for Health and Medical Research).

Email: elim.hong@inserm.fr

4: School of Physics, Trinity College, Dublin, Ireland.

Email: stefan.hutzler@tcd.ie

5: School of Physics, Trinity College, Dublin, Ireland.

Email: irannezhad.a@gmail.com

6: Aquatic facility, Pierre and Marie Curie Campus, Sorbonne University, Paris, France.

Email: abdelkrim.mannioui@upmc.fr

7: School of Physics, Trinity College Dublin, Ireland.

Email: peter_richmond@ymail.com

8: Institute for Theoretical and High Energy Physics (LPTHE), Pierre and Marie Curie campus, Sorbonne University, Centre de la Recherche Scientifique (CNRS). Paris, France.

Email: roehner@1pthe.jussieu.fr

9: Aquatic facility, Pierre and Marie Curie Campus, Sorbonne University, Paris, France.

Email: stephane.tronche@upmc.fr 


\section{Abstract}

Infant mortality, by which we understand the postnatal stage during which mortality is declining, is a manifestation and embodiment of congenital abnormalities. Severe defects will translate into death occurring shortly after birth whereas slighter anomalies may contribute to death much later, possibly only in adult age. While for many species birth defects would be nearly impossible to identify, infant mortality provides a convenient global assessment. In the present paper we examine a broad range of species from mammals to fish to gastropods to insects. One of the objectives of our comparative analysis is to test a conjecture suggested by reliability engineering according to which the frequency of defects tends to increase together with the complexity of organisms. For that purpose, we set up experiments specially designed to measure infant mortality. In particular, we two species commonly used as model species in biological laboratories, namely the zebrafish Danio rerio and the rotifer Brachionus plicatilis. For the second, whose number of cells is about hundred times smaller than for the first, we find as expected that the screening effect of the infant phase is of much smaller amplitude. Our analysis also raises a number of challenging questions for which further investigation is necessary. For instance, why is the infant death rate of beetles and molluscs falling off exponentially rather than as a power law as observed for most other species? A possible research agenda is discussed in the conclusion of the paper. 


\section{Contents}

\section{Introduction}

\section{Infant mortality in humans}

Infant versus adult mortality

How can one draw information on congenital defects from infant mortality?

Infant mortality across non-human species: framework

Cross species similarities in prenatal and neonatal growth

Relationship between neonatal death rates and the functions that must be switched on

Broad overview for mammals, crocodilians and birds

Infant mortality for zebrafish

Slope of the power law fall

Conjecture for predicting the infant mortality peak of fishes

Infant mortality of beetles and molluscs

Beetle

Mollusc

Infant mortality for rotifers

Distinctive features of rotifers

Design of the experiment

Tentative interpretation of differences in mortality rates

\section{Conclusion}

Main observations and questions

Agenda for future comparative research

Appendix A: Previous studies of the mortality of rotifers

Main features

Comments about variability

What conclusions can one draw with respect to infant mortality?

Appendix B: Methodology of the rotifer experiment

Procedures

Operational definition of death

Appendix C: Correct estimate of the initial death rate 
This paper is the second leg of an exploration in three parts; the two others are Bois et al. (2019a,b). Despite the connections, the three papers can be read independently from each other.

\section{Introduction}

Currently, the standard view 1 is that congenital anomalies should be attributed to genetic or environmental factors. Genetic factors can be in the form of genes inherited from the parents or somatic mutations occurring during pregnancy. Environmental factors may refer to chemicals (e.g. alcohol, nicotine and drugs) or infectious agents (e.g. parasites, bacteria and viruses) impacting the body of the mother during pregnancy. In Bois et al. (2019a) we introduced a third source of congenital anomalies. It consists in random fluctuations of the "manufacturing" process. It is by purpose that we use the term "manufacturing" to establish a parallel with reliability engineering. As real manufacturing outputs always differ slightly from design values, the challenge of engineering is to combine all such defective parts in a way which ensures that the end-product works.

A natural idea is that manufacturing defects are particularly crucial in components of complex systems which involve several steps and require high accuracy. In Bois et al. (2019a) we give several examples of that kind. For instance, any defect in heart valves (e.g. 2 leaflets instead of 3) may imperil the good working of the heart.

Conversely, one would also expect that organisms with a simple structure will be less impacted by output defects. Here is an example. If because of their small size some organisms do not need to be equipped with a circulatory system comprising a heart one would expect a reduced rate of infant mortality. This conjecture is indeed confirmed by the case of the rotifers which will be examined below.

Our study develops through the following steps.

(1) First, we recall the shape of infant mortality in humans. It is an excellent starting point because very detailed data are available. In addition it defines a pattern which is more or less the same in other species as will be seen through various graphs presented in this paper.

(2) Then, we turn to fish. In this case infant mortality is well known both through studies done in aquaculture farms and through our own experiments. One intriguing fact is that the exponent of the power law which describes the fall of the death rate is around 3 rather than around 1 as for mammals. Another open question is the length

\footnotetext{
${ }^{1}$ As an illustration of this position one can cite the following statement: "Birth defects may result from genetic disorders, exposure to certain medications or chemicals, or certain infections" (excerpt from the introduction of the Wikipedia article entitled "Birth defects").
} 
of the decrease phase. One knows that it lasts several months but one does not know its exact duration.

(3) For molluscs and beetles there is another surprise in the sense that the death rate does not fall like a power law but rather as an exponential.

(4) Continuing our progression toward ever simpler organisms, we describe the experiment done for rotifers. It turns out that, as expected, the amplitude of the screening is rather limited.

(5) Finally, in our conclusion we propose an agenda for further investigations.

\section{Infant mortality in humans}

\section{Infant versus adult mortality}

Human infant mortality has already been discussed in Bois et al. (2019a). Here we wish to recall it for the purpose of comparison with subsequent cases and we do that with a graph which has a dual age scale, first logarithmic then linear, so as to emphasize the distinction between the infant mortality phase and the subsequent wearout phase.
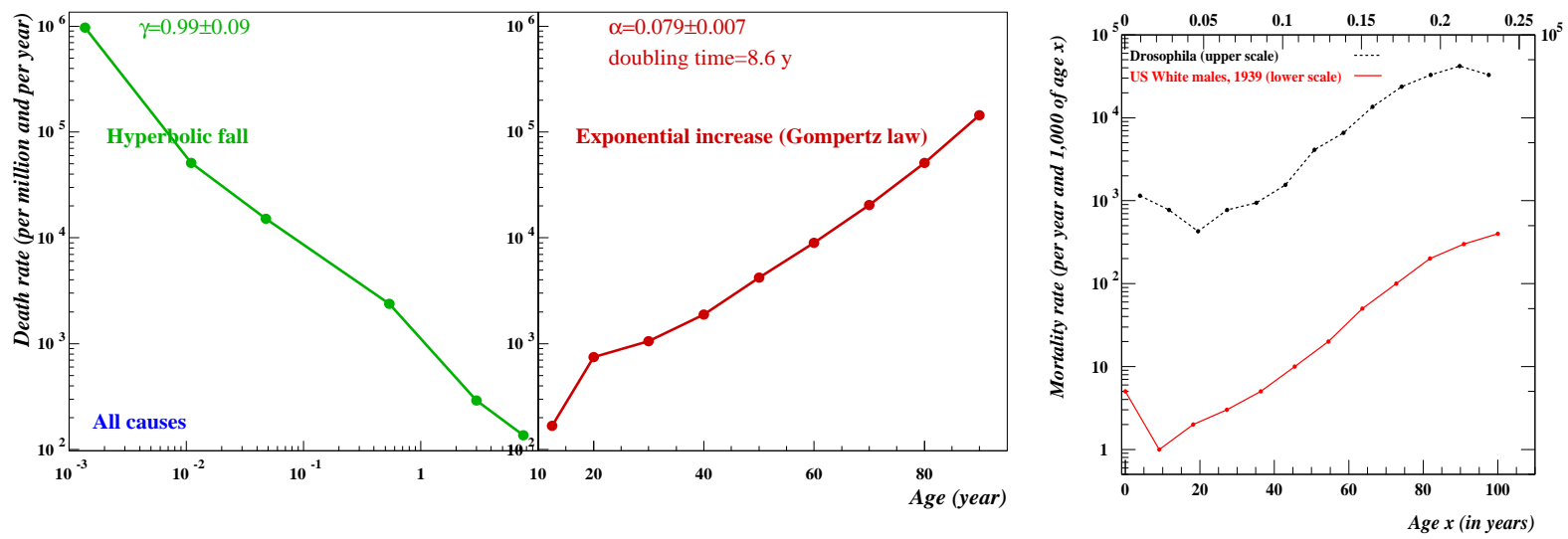

Fig. 1a,b Infant versus adult mortality rates for humans and drosophila. Fig.1a describes the two phases of human mortality. The data are for the United States over the period 1999-2016. Between birth and the age of 10 the infant mortality rate falls off as a power law: $\mu_{b}=A / x^{\gamma}$ where the exponent $\gamma$ is usually of the order of 1. Fig.1b shows a parallel with similar phases in Drosophila melanogaster. Source: Wonder-CDC data base for detailed mortality, Myio et al. (2004), Strehler (1967).

The graph of Fig.1b shows that, as for humans, there are two similar phases for the common fruit fly Drosophila melanogaster.

How can one draw information on congenital defects from infant mortality?

For each of the species investigated in this paper we will measure the age-specific infant mortality rate. Obviously, we will not be in a position to measure mortality rates for specific birth defects. This leads us to ask whether, from the "all causes" rate, one can derive information about malformations. Intuitively, it seems fairly 
evident that one cannot hope to derive detailed information about individual defect cases. However, in the discussion which follows it will be seen that the "all causes" curve in a sense summarizes some salient features of the curves for main classes of malformations.
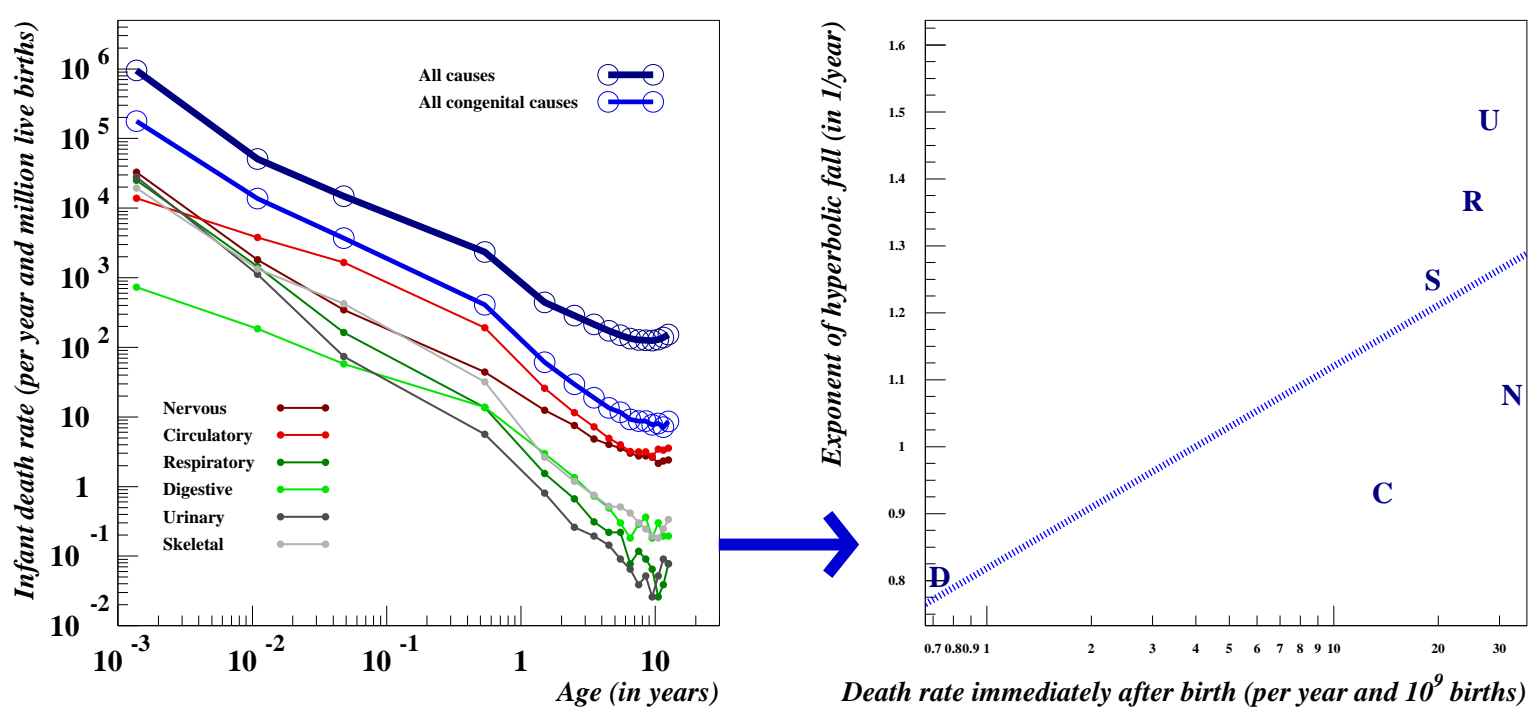

Fig. 1c,d Mortality from "all causes" versus mortality from main classes of birth defects. Fig.1c describes mortality rates for several classes of congenital defects. Usually, for animal species one can only measure the mortality from "all causes". However, Fig.1c shows that in the first year the curves for "all causes" and for "all congenital causes" are parallel. Moreover, Fig.1d shows that there is a connection between mortality rates at birth and the slopes of the regression lines of subsequent rates (which are identical to the exponents $\gamma$ of the hyperbolic falls). The correlation is 0.74 with a $95 \%$ confidence interval of $(-0.18,0.97)$. Due to the small numbers of data points it makes sense to rather choose a confidence level of 0.80 in which case the confidence interval becomes $(0.20,0.93)$. With the same confidence level the slope of the regression line is $a=0.14 \pm 0.08$. The capital letters refer to the 6 classes of defects listed in Fig. 1c. Source: Wonder-CDC data base for detailed mortality.

Fig.1c represents the curve (let us call it $\mathrm{C}_{\text {all }}$ ) of the death rates for "all causes" and similar curves for the main components of congenital malformations. One sees that the successive segments of $\mathrm{C}_{\text {all }}$ are parallel to the corresponding segments of the largest of the components. This is particularly clear in two cases: (i) the first segment is parallel to the corresponding segment of the nervous system curve, (ii) the third segment is parallel to the corresponding segment of the curve of the circulatory system. This property is due to the fact that on this log-scale graph "smaller" actually means "almost negligible". Therefore, it is not surprising that the range of the slopes of the segments of $\mathrm{C}_{\mathrm{all}}$, which (in absolute value) is $0.76-1.62$, reflects the range of the slopes of the regression lines of the various components, which is $0.80-1.49$. The averages are also almost the same: 1.10 for the first against 1.13 for the second.

Fig.1d shows another regularity. It says that the curves which start the highest fall 
the fastest 2 .

A quick argument can make this understandable. When, for a given defect, the death rate immediately after birth is high, all such defects will be eliminated rapidly with the result that subsequent death rates for this defect will become small, hence a steep descending curve.

This is a rather simplistic argument, however (which is why we called it a "quick argument") for it ignores the fact that defects of a given sort are not all of same severity. There are very severe defects (let us call them "first rank" defects) which lead to death in a very short term, e.g. for humans whithin one day and slight defects (let us call them "last rank" defects) which lead to death at longer term, e.g. for humans from months to years after birth. Needless to say, between first and last rank defects there may be a broad range of intermediate degrees of severity. As the distribution of each defect in terms of severity is a purely empirical matter, it is hardly possible to make a general argument. Nevertheless, the correlation displayed in Fig.1d suggests that, whatever the details, things seem to work in a way that is consistent with the previous argument.

This regularity can be summarized by saying that knowledge of the death rate at birth allows us to predict subsequent death rates in the early part of the infant mortality phase.

\section{Infant mortality across non-human species: framework}

\section{Cross species similarities in prenatal and neonatal growth}

From plants to fish, to birds, to mammals there is a bewildering diversity of living organisms. Yet, if one leaves aside the arthropods 3 there is a deep similarity in the mechanism through which a new organism starts its life. It begins as a tiny onecell embryo which divides and grows. In the case of a plant the energy required for the growth process is generated from the food reserve contained in the grain and the oxygen which diffuses through the grain's envelope. For the eggs of fishes and birds it is basically the same process. For mammals nourishment and oxygen come through the umbilical cord. After germination, hatching or birth the new organism must become autonomous in the sense of relying only on the resources (oxygen, carbon dioxide, light, food, and so on) available in its environment.

\footnotetext{
${ }^{2}$ If one interprets the death rates as velocities, their changes with age are accelerations. Translated into this language, the previous statement becomes: "The diseases which have a high initial velocity experience a strong deceleration".

${ }^{3}$ Because they have a rigid exoskeleton their development involves widely different instar stages. This mechanism leads to a different postnatal death rate pattern. The death rate is still decreasing but its shape is no longer hyperbolic (see Berrut et al. 2016, Fig.11). Note that the arthropods are a very large group which includes all insects and crustaceans. Although these two classes are the commonest, other well known arthropods are spiders, centipeds and millipeds. Altogether the arthropod group includes about $80 \%$ of all described living animal species.
} 
The similarity of these mechanisms leads to the question of whether or not postnatal death rates follow a general pattern.

\section{Relationship between neonatal death rates and the functions that must be switch- ed on}

Fig.1a,b shows that for humans birth is marked by a huge spike. On the contrary for the eggs of fishes hatching is not marked by any significant peak (see below Fig.3b); in fact there is a peak but it occurs several days after hatching. How can one explain that?

In order to survive an animal must be able to find food and to digest it. For animals feeding on preys finding food implies several challenges. (i) Identification of the foodt. (ii) Moving to where it is located. (iii) Swallowing and digesting. For animals (such as rotifers) which get food by filtering the water the process is reduced to step (iii). In addition mammals and birds need to regulate their body temperature. All species also need to develop their immune system but this is usually postponed for later because the yolk contained in the egg provides antibodies which afford protection as does also the milk provided by the mother in the case of mammals.

Coming back to the comparison of newborns and fish larvae is there a great difference in the functions that must be switched on?

The main difference is probably that humans need to breathe whereas larvae receive their oxygen by diffusion through their skin until about three weeks after hatching. Another difference is that human newborns must be able to suck and digest the mother's milk whereas the blood of larvae is channeled through their yolk sack which means that no digestion process is involved. Then, when the yolk sac is depleted fishes have to find their food by themselves. As an illustration one can mention that in zebrafish the duration of the yolk sac is about 6 days. Observation shows that once the sac is empty if no food is forthcoming a zebrafish can survive some 3-4 days. Based on the previous argument one expects a death rate peak some 10 days after fertilization, a prediction that is indeed confirmed by observation. As a matter of fact, for all species for which data are available observation showed a death rate peak occurring in the expected time interval. For salmons, due to their huge yolk sac, the death rate peak occurs some 60 days after fertilization.

\section{Broad overview for mammals, crocodilians and birds}

Fig.2 shows data for various animals kept in zoos. In spite of the fact that the data are fairly sparse one can learn two things from this graph.

\footnotetext{
${ }^{4}$ It is interesting to note that through a series of experiments the biologists Andres Carrillo and Matthew McHenry (2015) were able to show that there is a learning curve for the ability to identify and catch a prey.
} 




Fig. 2 Infant versus adult mortality rates for animals kept in zoos. The graph displays three main features, (i) Over the first $10 \%$ of the life spans, the death rates are decreasing which means that this age interval is included in the infant mortality phase. (ii) The 3 data points in the infant mortality phase (which correspond to the age $t$ of one week, one year and two years) are compatible with an hyperbolic fall of the form: $\mu=A / t^{\gamma}$. The estimates for $\gamma$ corresponding to the 7 classes are written in front of the labels. Crocodilians stand out as an exception both in terms of $\gamma$ as in death rate level. The average of the 6 other classes is $\gamma_{m}=1.02 \pm 0.06$. Note that whereas the normalization of ages is useful for drawing the graph clearly, the estimates of $\gamma$ are independent of this normalization. (iii) Whereas the data for infant mortality display an homogeneous and orderly pattern, the data for adult mortality are highly variable and species dependent. Note that the righthand side of the horizontal age axis is rather symbolic. The normalization is based on a column of the source labelled "Life expectancy at 15 years". As each class contains several species, the magnitude of the estimates are somewhat arbitrary (but the succession of the ages is meanigful). That is why some data points are beyond 1. Source: Kohler et al. (2006). The different classes contain several species with main components as follows: Apes: gorilla, orangutan; Small primates: ring-tailed lemur, ruffed lemur; Carnivores: lion, tiger, cheetah; Hoofstock: North American bison, Arabian oryx; Crocodilians: American alligator, Johnston's crocodile; Ratites: greater rhea, common emu; Raptors: bald eagle, king vulture.

(1) The infant death rates are much more regular and uniform than the late-age death rates (we come back to this point below). They are also much higher.

(2) With respect to the exponent $\gamma$, of the 7 subgroups there is one which emerges as quite different, namely crocodilians. The average of the 6 others is (with probability level 0.95$): \gamma_{m}=1.02 \pm 0.12$. The case of the crocodilians is particularly spectacular because they have not only a lower slope, namely $\gamma=0.52$ but also a much lower overall death rate. 
Why do the mid/old age rates show such a high dispersion? Two answers come to mind.

It may be due to the well known fact that aging is highly disease dependent and diseases have of course high variability. The point is developed in Jones et al. (2014).

A second reason which may contribute significantly to the disperse nature of the aging process is the frequent relocation of zoo animals between zoos. A look at the ISIS (International Species Information System) records (from which the data were extracted) shows that most of the animals do not spend their whole life in the same zoo; instead they are repeatedly loaned (or sold) by one zoo to another. This may be disturbing for the animals but it raises also a book keeping problem. In the paper by Kohler et al. (p. 429) it is reported that even highly visible animals such as gorillas "are assigned multiple identification numbers in various regions around the world". What occurs for gorillas is also likely to occur for other species. The only difference may be that for low profile species the inaccuracies in the records remain unnoticed.

More detailed infant mortality data for various species of primates can be found in Berrut et al. (2016, p. 417). The exponents $\gamma$ of the power laws are comprised between 0.9 for silvery marmoset and 1.4 for common marmoset. In this paper it has been checked that the data from Kohler (2006) are consistent with the more detailed data of Pouillard (2015).

\section{Infant mortality for zebrafish}

The zebrafish (Danio rerio) is used as a model organism especially for developmental and genetic analysis. Among its advantages one must highlight its small size (ca. $3 \mathrm{~cm}$ at adult stage) and its transparent eggs and body during embryo and larva development.

\section{Slope of the power law fall}

The figures 3 and 4 show two things:

(i) The infant death rate falls off as a power law $y \sim 1 / x^{\gamma}$ (ii) $\gamma$ is of the order of 3 or 4. Two other cases are shown in Fig.5a,b which also give a $\gamma$ of the order of 3. This is contrast with the case of mammals for which $\gamma$ is around 1 (see the values given for various primates in Berrut, 2016). For crocodilians, Fig.2 gave a value of 0.52. Whereas the values for mammals relies on the study of various species, for fish and crocodilians more data would be welcome. In other words, before trying to find an interpretation the previous values need to be confirmed.

Can one say that such small fishes are simpler than the mammals considered previously?

The difference is more quantitative than qualitative. Fish are vertebrates which 

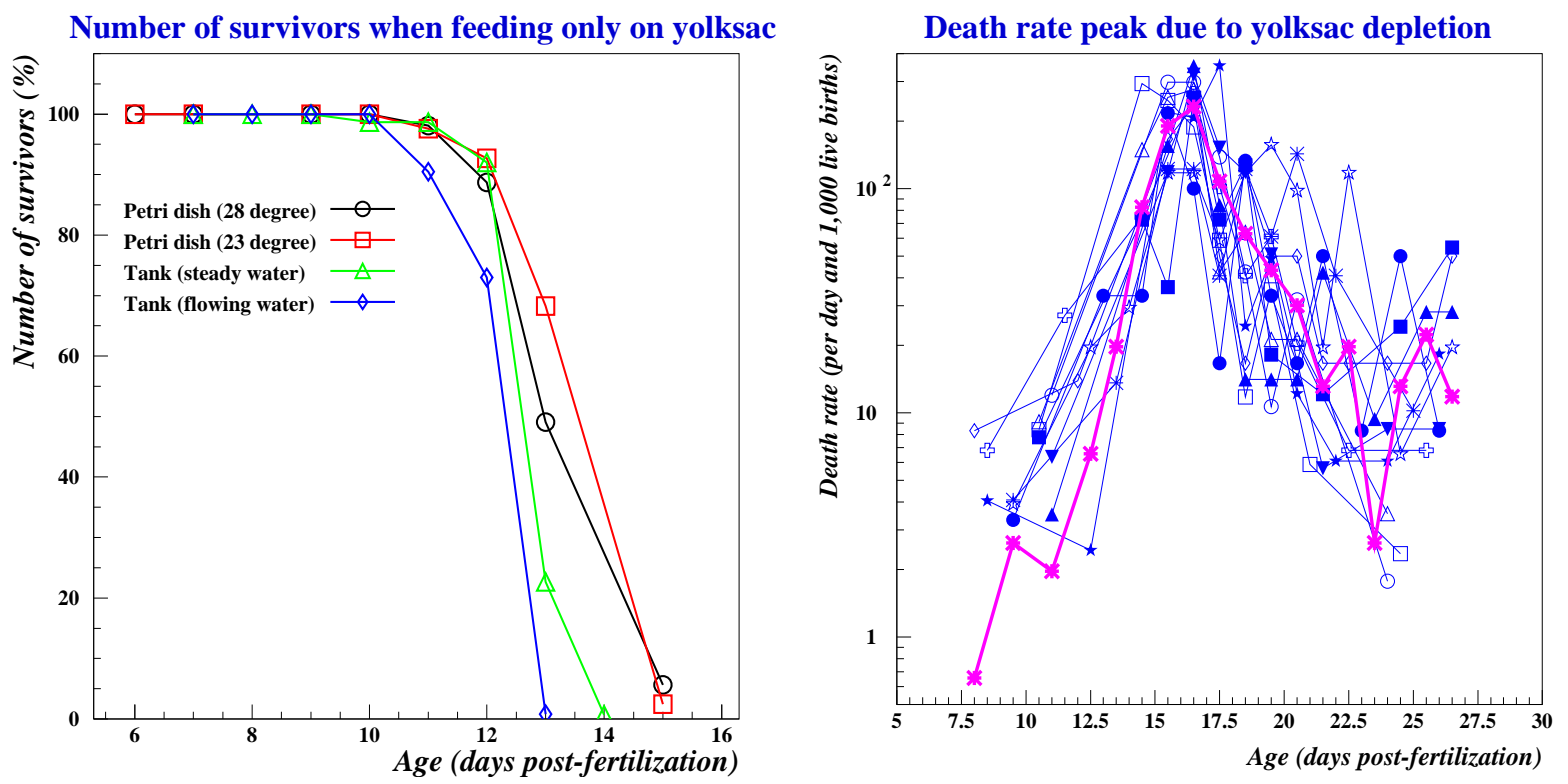

Fig. 3a,b Mortality effect of yolk sac depletion. Left: No food given. It can be seen that different conditions had only a small influence on the decrease of the population. Basically, the population fell faster when conditions required a greater energy consumption. Right: Food was given after day 5. Initially there were 12 samples each with about 75 larva which means a total population of about 1,000. The coincidence between the depletion of the yolk sac and of the mortality peak suggests that the latter is due to the fact that some larvae were not able to feed on the external sources of food. The thick line curve in red is the death rate of the total population; note that this rate is somewhat different from the average of the death rates of the 12 samples. Source: The experiments were performed in the spring of 2016 at the Aquatic facility, Pierre and Marie Curie Campus.

means that like mammals they have a spinal column and the same overall organization. At the end of its development in the egg the zebra embryo has 25,000 cells (Kobitski 2015) which is about one million times less than the 26 trillions of a newborn baby.

As already explained, from the perspective of infant mortality the important novelty of fish is the existence of the yolk sad5.

\section{Conjecture for predicting the infant mortality peak of fishes}

Apart from the case of zebrafish documented above, similar data for the early death rate of larvae are given in Berrut et al. 2016).

The basic rule is that the relative size of the yolk sac conditions the moment of occurrence of the post-hatch death rate peak. This is illustrated schematically in Fig.6. However, to our best knowledge, so far there has been no systematic study of this effect for a substantial number of fishes. That is why the graph is presented as a conjecture. Note that one expects a different relationship for species (e.g. the red-fish Sebastes mentella and Sebastes fasciatus considered in Berrut et al. 2016)

\footnotetext{
${ }^{5}$ Around 5 weeks in the development of a human embryo a yolk sac can be seen. As in fish it is the earliest source of nutrients for the developing fetus. However, after 8 weeks the yolk sac degenerates and disappears
} 

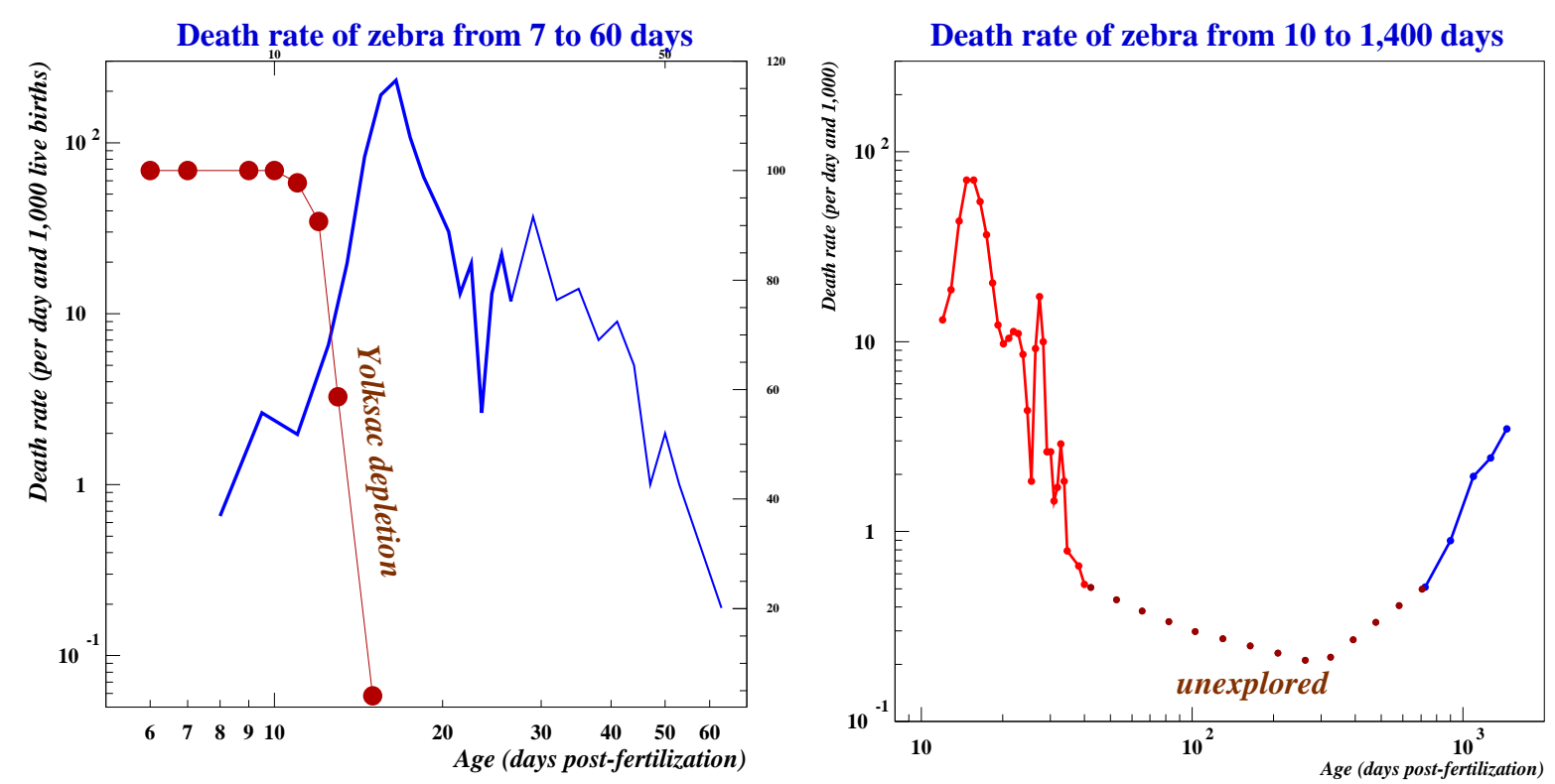

Fig. 4a,b Mortality profile of zebrafish from birth to senescence. Left: The curve with the big dots is taken from Fig.3a and shows the evolution of the population when no food is given (right-hand side scale). The experiment of Fig. $3 \mathrm{~b}$ was extended to 60 days; this part of the curve is drawn with a thinner width because after 40 days in each sample the numbers are reduced to a few dozens. A linear regression for the falling part of the curve gives a slope, that is to say an exponent of the power law $y \sim 1 / x^{\gamma}$ equal to $\gamma=4.5$. Right: On average zebrafish can live 3 to 4 years. There are two phases (infant and aging) separated by a transition. During the infant phase the death rate decreases as a power law whereas in the aging phase it increases as an exponential. For the falling part of the curve: $\gamma=4.2$. The dotted line is a section of the mortality profile which, to our best knowledge, has not yet been explored. It would be interesting to know the timing of the transition and at which death rate level it occurs. Note that the right-hand side of this graph cannot be directly compared to Fig.1a because here the entire age axis is logarithmic. Sources: Left: The experiment was performed in the spring of 2016 at the Aquatic Facility, Pierre and Marie Curie Campus. Right: Infant mortality: The experiments were done at the IFREMER laboratory in Rennes (France) between November 2011 and February 2013 (private communication). It can be observed that the left and right data are consistent with one another. For the senescent mortality the source is Gerhard et al. 2002.

for which fertilization occurs internally.

\section{Infant mortality of beetles and molluscs}

These cases are interesting because the shape of the infant death rate is not a power law but rather an exponential. Taken alone the beetle case may suggest that the difference comes from the fact that the graph covers only the adult stage. However, this explanation is contradicted by the mollusc case which features also an exponential fall in spite of the fact that there is a unique stage of development.

\section{Beetle}

As for many other insects, the development of beetles comprises 3 stages.

(1) The larva stage which follows the transition from egg to larva.

(2) The pupa stage which follows the transition from larva to pupa. 

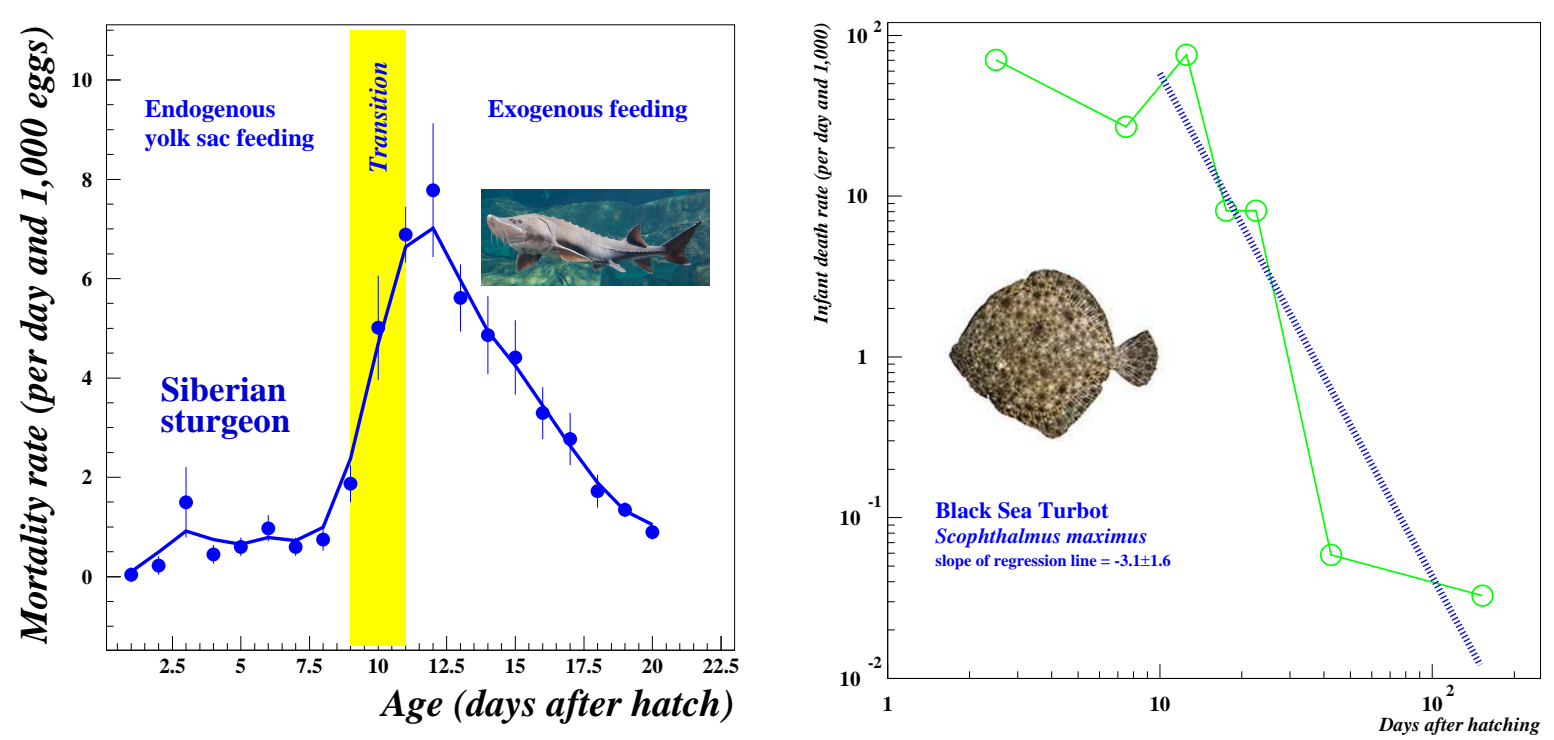

Fig. 5a,b Mortality rate of sturgeon and for Black Sea Turbot up to the age of $\mathbf{1 5 0}$ days. The left-hand side graph has not been drawn in log-log scales for reason of clarity. However, when fitted to a power law the descending part of the curve turns out to have an exponent $\gamma=-3.9$. For an accurate determination of the falling phase the Black Sea turbot graph has the advantage that the survivorship data extend from hatching up to day 246; this allows us to compute the death rate at age $(60+246) / 2=153$ days. It appears that the mortality falls off as a power law with exponent: $\gamma=-3.1 \pm 1.6$ (we gave the error bar explicitly because here there only few data points). Source: Sahin 2001.

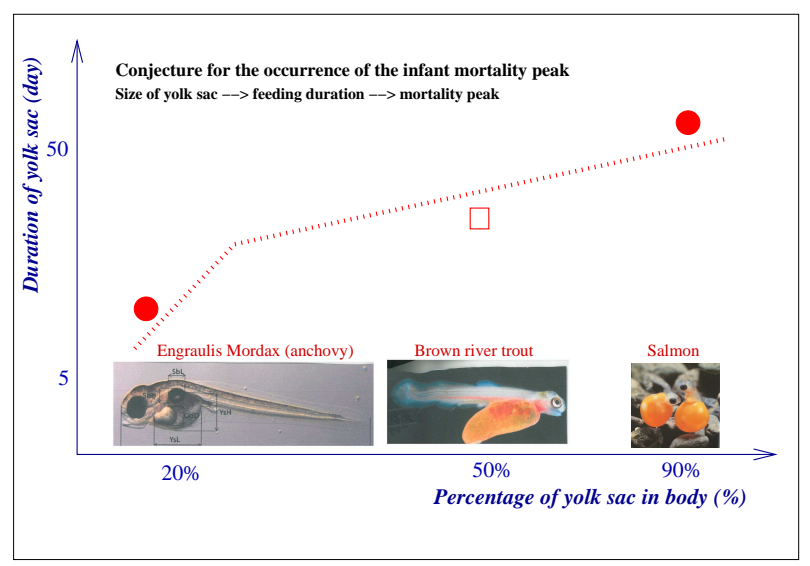

Fig. 6 Timing of the infant mortality peak for fish larvae derived from the size of their yolk sac. The graph schematically describes the relationship between the relative size of the yolk sac (with respect to the whole body at birth of the larva) and the duration of the endogenous feeding phase ensured by the yolk. Because precise data are available only in a few cases the exact shape of the relationship is still a conjecture. One expects a higher slope for short durations because in the first few days after hatching the larvae remain fairly motionless which means that a given amount of yolk can provide longer subsistence than later on when the larvae swim and expense more energy. The present graph is for species with external fertilization; one would expect a different relationship when fertilization and hatching occur internally. Sources: The yolk sac durations for the anchovy and salmon are taken from Berrut et al. 2016. The pictures are from Internet; note that the first picture is for a species similar to Engraulis Mordax.

(3) The adult stage which follows the pupa stage.

Unlike previous graphs, Fig.7a shows the life span after hatching after emerging 
from the pupa stage.

According to Pearl $(1941, \mathrm{p} .6,8)$ the total length of the larva and pupa stages of the flour beetle is about 50 days, whereas the median life-time in the adult stage is 200 days.

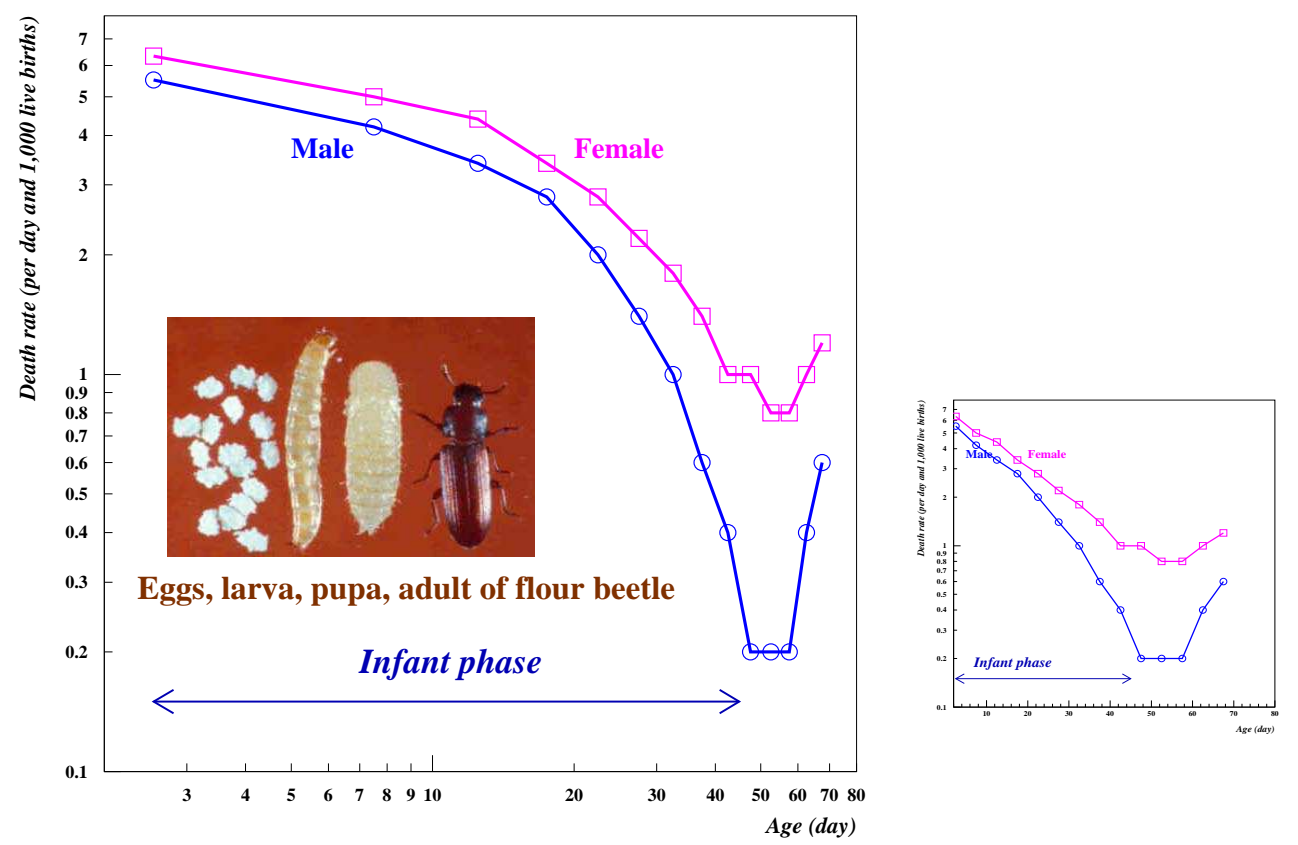

Fig. 7a,b Infant death rate of a flour beetle (Tribolium confusum). Left: log-log plot. Right: lin-log plot. In other words, the fall is almost exponential. Unlike previous cases, this curve does not show the life span after hatching but after emerging from the pupa stage. This stage follows a previous stage as a larva as illustrated in the insert. Note that the median life durations of males and females are 171 days and 210 days respectively. Thus, the transition between decline and increase of the death rate occurs at $28 \%$ and $26 \%$ respectively of the median life spans. This is a longer infant mortality phase than the $10 \%$ to $14 \%$ observed for mammals. Source: Pearl et al. (1941, p.8,13-14). Note that in 1945 a follow up paper was published which is about Tribolium madens.

It would probably help our understanding to know and be able to compare the infant mortality curves for the three successive stages.

\section{Mollusc (slug)}

Whereas Tribolium confusum is an insect belonging to the Coleoptera order, Agriolimax agreatis belongs to the class Gastropoda within the phylum Mollusca. Although their external aspects are very different their internal organizations are fairly similar. For instance both have what is called an "open circulatory system" in which the liquid which plays the role of blood is pumped into a cavity which contains the main organs which need to be supplied with oxygen. Perhaps it is for this kind of reason that in both cases the infant death rate falls off like an exponential (Fig.8a,b)

With its sophisticated heart (which includes 4 chambers) and its network of arteries and veins the circulatory system of mammals is fairly fragile. The arteries may be 



Fig. 8a,b Infant death rate of mollusc (Agriolimax agreatis). As in Fig.7a versus 7b, Fig.8b differs from Fig.8a by the scale of the horizontal axis. Source: Pearl et al. $(1935, p .62)$

blocked by blood clots and a insufficient supply of oxygen may stop the heart. Once the heart stops working, death follows within a few minutes. The simpler circulatory system of Coleoptera and molluscs is probably more resilient. If confirmed, this feature might explain why in those cases the fall with age of the infant mortality is smaller than for humans. In the case of mollusca the death rate is divided by only a factor 2. A similar case not represented here but for which data are given in Pearl et al. (1935) is the insect Blatta orientalis for which infant mortality also falls by a factor of two.

\section{Infant mortality for rotifers}

Apart from the measurement of zebrafish infant mortality (reported above) we have also set up an experiment to estimate the infant mortality of rotifers.

The rotifer Brachionus plicatilis is a species that is much used in biological research, including evolution (Declerck et al. 2017, Tarazona et al. 2017), ageing (Johnston et al. 2016) or ecotoxicology (Snell et al. 1995).

\section{Distinctive features of rotifers}

The $B$. plicatilis that we study in this section are smaller and simpler anatomically than the organisms tested previously. With an adult size of about $0.25 \mathrm{~mm}$, they are 100 times smaller than the beetles which were the smallest organisms described so far. They are also simpler in the sense that they have a constant number of about 1,000 cells 6 and do not need a heart or lungs because of their small size which allows them to rely on diffusion for oxygen supply throughout their whole body.

For our experiment an important point is their lifespan. In the literature the estimates

\footnotetext{
${ }^{6}$ The number of cells increases until adult age and then remains constant
} 
range from 3.3 days (Gopakumar et al. 2004) to about 14 days (Korstad et al. 1989, Snell et al. 2016, Sun et al. 2017). How can one understand such a broad range? Usually, it is explained by the fact that there are many parameters to be taken into account, for instance: temperature, salinity, type of food, strain. The following remarks should help to reduce the variability of lifespan estimates.

(1) The lifespan should not be estimated by the time it takes for the population to vanish but rather by the time to 50\% survivors. Why? The survival of the last 2 or 3 individuals is a "small $n$ experiment open to large fluctuations. In addition the age of the last survivor depends upon the size of the sample. In a sample of 1,000 French people there may not be a single centenarian but in a sample of 10,000 there will be about 3 . The previous $50 \%$ threshold can be replaced by a smaller one but only if the initial size $\left(n_{0}\right)$ of the sample is sufficient. If $n_{0}=100$ a threshold of $10 \%$ will lead to a final group of only 10 , a small number which may give rise to sizeable statistical fluctuations. In Table A1 we give lifespan estimates based on a threshold of 50\%; their average is: 8.5 days.

(2) Needless to say, one should avoid small samples. The results of Gopakumar et al. (2004) are based on samples of only 10. Of the 6 experiments mentioned in Table A1 five have a $n_{0}$ smaller than 200.

(3) Finally, it should be observed that the effects on lifespan of temperature and salinity are rather limited. According to the data recorded in Gupakumar et al. (2004) a $100 \%$ salinity increase diminish the lifespan by only $13 \%$ and a $85 \%$ increase in temperature (from $20^{\circ} \mathrm{C}$ to $38^{\circ} \mathrm{C}$ ) increases the lifespan by only $1 \%$ (which is certainly smaller than the experimental error bars).

Even the diet may not be as crucial as may seem at first sight. It is true that Korstad et al. (1989) observed lifespans ranging from 5 to 14 days depending on the diet. Interestingly however, a 5-day survival was also observed (Garcia-Roger et al. 2006) when no food is given. This suggests that the algae used by Korstad which gave the shortest lifespan was simply inappropriate for B. plicatilis.

For these reasons one expects less congenital defects and therefore less infant mortality. It is likely (as already observed in Bois et al. 2019a) that the two effects, namely elimination of defective organisms and wearout, are at work simultaneously. Hence, one wonders whether the reduced infant effect will still be visible or whether it will be overcome and masked by the wearout effect. In other words, will there be an age interval where the mortality is declining? This was our main interrogation. The answer is "yes".

\section{Design of the experiment}

The experiment is fully described in Appendices B and C. It involved the following steps. 




Fig. 9 Infant death rate of rotifers (Brachionus plicatilis). The graph summarizes the results obtained for 17 cohorts. No selection was performed in the results which means that even the trials which led to "surprising" outcomes (for instance large death numbers fairly early in the aging phase) were included. Each trial involved several hundred rotifers (the range was from 500 to 1,500). When adjusted to a power law the descending part of the curve corresponds to an exponent of 0.33. Source: Experiments conducted at the "Aquatic Facility" of the "Pierre and Marie Curie" campus in January-April 2019.

(1) The basic requirement was to prepare a large set of newly hatched rotifers. However, "large" and "newly" are two conflicting requirements. Why?

Because of the variability in the duration of the embryonic phase, the process of hatching for a large number of individuals will be spread over one or two days. Hence, if one allows hatching to go on for only one hour the ages of the hatchlings will be defined with an accuracy of \pm 0.5 hour but on the other hand one will get only $1 / 24=4 \%$ of the total number of hatchlings.

This conflict can only be made good by using as many eggs as possible. Typically, in each separate trial we used of the order of 8,000 eggs 7 . For a hatching duration of 3 hours this produced about 1,000 hatchlings.

(2) In the measurement process there is a similar conflict between the number of deaths and the time interval between successive measurements. The reason is obvious. If one waits $6 \mathrm{~h}$, there will be many deaths but their ages will have a broad $\pm 3 \mathrm{~h}$ uncertainty. On the contrary if the measurements are done every $2 \mathrm{~h}$, the ages will be defined $\pm 1 \mathrm{~h}$ but there will be few deaths and therefore large statistical fluctuations.

\footnotetext{
${ }^{7}$ The number of eggs in a sample can be estimated by the area that they occupy in the field of the microscope when they are closely packed together without overlap.
} 
We adopted the following schedule. During the first 12 hours measurements were made every 3 hours from morning to evening, then the next ones were made $12 \mathrm{~h}$ apart on the following morning and evening, and then every $24 \mathrm{~h}$ for two or three days.

Each measurement implies the following operations.

(1) One scans line by line the content of a petri dish ( $9 \mathrm{~cm}$ in diameter).

(2) All non-swimming rotifers are examined to decide whether or not they should be considered as dead (see the discusssion of this point in Appendix C).

(3) One removes all dead rotifers. The density of the swimming rotifers should not be too high for otherwise it is almost impossible to remove the dead without also removing some of the living.

The graph of the mortality rate resulting from a series of measurements is shown in Fig.9. We would be happy if its overall shape could be confirmed by observations made independently by another team. In physics every significant observation is usually re-tested for confirmation by several other teams using alternative methods and devices.

\section{Tentative interpretation of differences in mortality rates}

We consider infant mortality for (a) fish, (b) mammals and (c) crocodilians. In all three cases the infant death rate decreases as a power law which means that on a $\log -\log$ plot the death rates are straight falling lines. However, their slopes (which correspond to the exponents of the power laws) are very different: about 3.5 for fish, 1 for mammals and 0.5 for crocodilians 8 .

Fig.10 shows the slopes of infant mortality for several classes of species considered earlier. What does the comparison tell us?

- On account of their physiological similarity one is not surprised that humans and primates have the same slope. The fact that the line for primates is much higher is also understandable for young primates are known to have a high mortality in captivity conditions.

- The cases of fish and crocodilians look very different. For the sake of simplicity we assume that in each class of species there is only one kind of defect, a different one in each class. The high level and steep decrease of the line for fish indicates a kind of defect which is lethal within a short time; then, as these individuals get rapidly eliminated the mortality falls rapidly. On the contrary, for crocodilians one expects slight defects leading to low death rates, hence a slow elimination of defec-

\footnotetext{
${ }^{8}$ The crocodilian class includes 1,914 American alligators (Alligator mississippiensis), 188 Johnston's crocodiles (Crocodylus johnstoni) 67 Cuban crocodiles (Crocodylus rhombifer).
} 


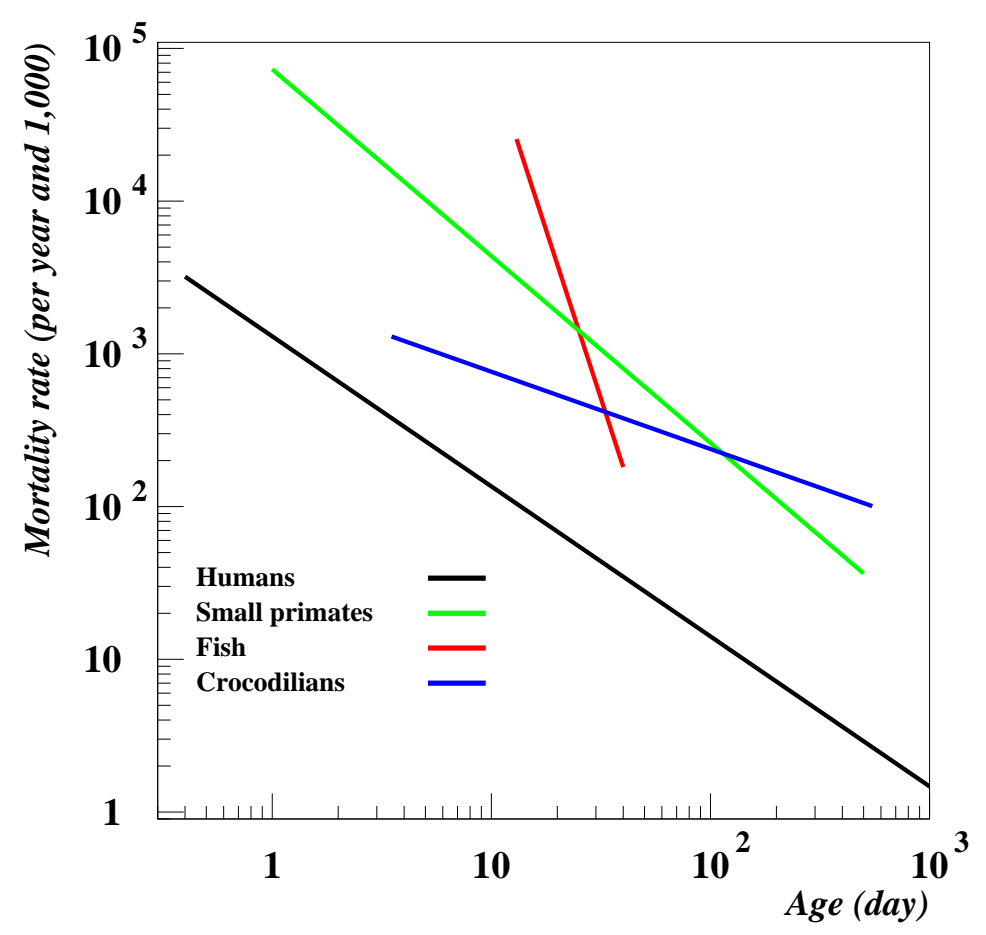

Fig. 10 Infant mortality in comparative perspective. We did not include the rotifers because their scale is different. Source: Data from previous graphs.

tive individuals. Thus, the mortality starts from a low level 9 and decreases slowly. In a general way, a low initial infant mortality means a slow elimination process and is therefore likely to be associated with a low slope of decrease.

\section{Conclusion}

Previous studies (including our own studies) did hardly attempt to compare infant mortality on the basis of complexity (say, number of cells and anatomical sophistication). This was the main objective of the present paper. The expression "anatomical sophistication" refers primarily to the number of vital organs. For instance, human newborn have a heart and lungs whereas zebra larvae have a heart but no lungs and rotifers (whether newborn or adult) have neither heart nor lungs.

How, then, do rotifers die? Although this remains largely an open question, one can imagine the following sequence of events. Rotifers do have a digestive system; if it is defective they will have little energy for swimming and rotating their wheels. Immobilization will prevent them from ingesting food therefore reducing even more their energy supply. An implication of this scenario is that for rotifers immobility foreshadows death.

\footnotetext{
${ }^{9}$ Naturally, the level of mortality at birth depends also upon the prevalence at birth of the defect that is to say the percentage of individuals who have this defect. In our argument we have implicitly assumed that the prevalence is of similar order in the two classes of species.
} 
Based on this qualitative argument one would of course expect rotifers to have a lower death rate at birth than humans 10 . Therefore, according to the empirical rule derived from Fig.1d, one would expect a lower exponent for rotifers than for humans. This is indeed confirmed by observation.

Naturally, one is tempted to apply the same argument to a comparison between humans and fish. This is done in Fig.10. The fact that a higher initial death rate leads to a higher exponent is again consistent with the rate-exponent rule of Fig.1d. However it remains to understand why the initial death rate of zebrafish is so high. For that purpose one would need mortality data for zebrafish for ages of about 6 months to one year.

\section{Main observations and questions}

A discussion of manufacturing defects (in the companion paper Bois et al. 2019a) revealed that, not surprisingly, there is a relation between the severity of a defect and the age at which it generates a peak in the curve of failure rates. The infant mortality rate of fish provides an illustration. Possible defects in the ability to transition from yolk sac feeding to prey catching are revealed by a massive peak in the death rate curve. More generally, any impairment in a transition will give rise to a mortality peak but to be clearly visible on the mortality graph the transition must occur in a sufficiently short time interval. Thus, because the transition from skin to gill oxygen uptake takes much longer than the yolk sac transition (Rombough 1993) it is hardly visible on the death rate curve.

A pending question that was raised (but not yet solved) by the previous comparisons is why in mammals and fish the infant death rate has a power law fall whereas for beetles and molluscs it is an exponential fall.

\section{Agenda for further comparative studies}

The selection of the species mentioned in the table below is based on two criteria.

(i) Development in liquid medium.

(ii) Ability to swim.

The first condition excludes the larvae of drosophila or other insects which develop on solid media11.

\footnotetext{
${ }^{10}$ This is difficult to check on the statistical data for two reasons. (i) The death rate of human newborn depends strongly on their age. At the age of 2 hours, the death rate is 100 times higher than at the age of one week (see Berrut et al. 2016, Fig.5a); therefore, depending upon the age taken as reference the comparison will lead to different conclusions (ii) The death rate at birth is usually defined per year and 1,000 newborn. In order to make a comparison between a rate for humans and the rate for a species $S$ one must convert 1 year in its equivalent for species $S$. The attempt made in Fig.2 for fairly similar animals (mostly mammals) showed that this is not easy but it becomes really very hazardous when $S$ is a rotifer species.

${ }^{11}$ The following pitfalls can be mentioned: By drilling holes into the agar the larva became invisible; they climb on top of one another which makes counting impossible; through its fast growth the yeast tends to recover the bodies of dead larvae and makes counting uncertain.
} 
Table 1: Selection of species appropriate for a comparative study of infant mortality

\begin{tabular}{lllccc}
\hline \hline Species & Description & $\begin{array}{c}\text { Number } \\
\text { of cells at birth }\end{array}$ & $\begin{array}{c}\text { Length } \\
\text { of adult } \\
\text { [cm, mm] }\end{array}$ & Lifespan \\
[year,day]
\end{tabular}

Notes: The species are ranked by decreasing size and complexity. All of them can grow in liquid medium and are able to swim. For zebrafish the Latin name is Danio rerio. As reported previously this organism has already been studied but we believe that greater accuracy can be obtained with larger populations. For unicellular organisms the lifespan is equivalent to the doubling time. 1 and 2 have already been described in the text. 3, 4 and 5 reproduce asexually through binary fission but 3 and 5 have also a form of reproduction which allows two individuals to exchange genetic material.

Sources: Burdett et al. (1986), Joe (2004).

The second criterium excludes organisms like yeast which cannot swim. Mobility is important because it gives a simple way for deciding whether an individual is alive or dead.

\section{Specification of birth and age for unicellular organisms}

How can one extend to unicellular organisms the notion of age-specific mortality rate? At first sight it might seem that unicellular organisms do not die. However, closer examination reveals that for bacteria such as E. coli or B. subtilis the size can be used as a proxi for age. After birth through binary fission the size of the organism increases until it reaches a critical threshold at which point a new binary fission starts (Walden et al. 2016). The analog of embryonic mortality will correspond to the time interval between the moment when the preparation of the division starts (the first step is usually the replication of the chromosone) and the moment when separation takes place.

The infant phase starts imediately after separation. It is likely that a small percentage of the new cells will not be viable because of some defect which occurred in the growth and division process.

As always, the cells with a major defect will die immediately whereas the cells with only a slight defect will die later. If this conjecture is correct one will observe a decreasing mortality rate from birth until a subsequent, but yet unknown, time.

It is this mortality curve that one wishes to record because it will give us global information about the variability and reliability of the replication process.

The starting point of our previous experiments with zebrafish and rotifers was a large 


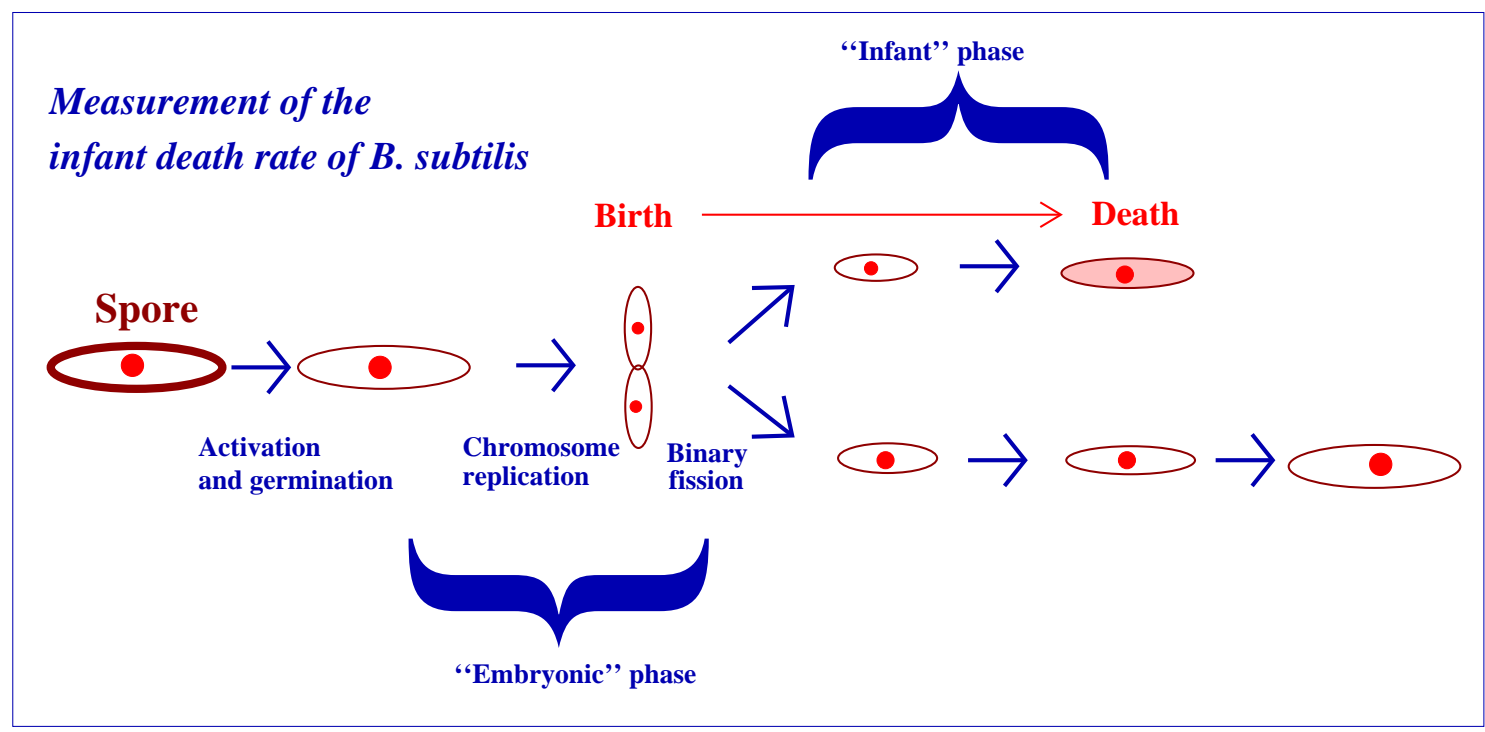

Fig. 11 Measurement of the age-specific infant mortality rate of $B$. subtilis. The red dot figures the single circular chromosome. Just to give an order of magnitude one expects an infant mortality rate of the order of $10^{-5}$ per hour and per 1,000 live "births", this last number being the magnitude of the failure rate of the process of human DNA replication. In appropriate conditions the activation and germination of the spore cells can take place in 3 or 4 hours. Note the increase in size of the chidren cells between birth and the adult stage which precedes the next division. Sources: Hosoya et al. 2007, Wallden et al. 2018.

set of eggs. What will be our eggs in the present case? In response to nutrient deprivation, $B$. subtilis forms dormant spores which can be reactivated by appropriate means. Thus, the experiment can be summarized as shown in Fig.11.

\section{Appendix A: Previous studies of the mortality of rotifers}

In this section we summarize and compare mortality results obtained in former observations. As can be seen immediately by a look at Fig. A1 they are characterized by a great variability. Broadly speaking this variability has two main sources. (i) Small samples (a few dozens is fairly common) result in large statistical fluctuations. (ii) The diet provided to the rotifers can have dramatic effects. This is illustrated in a paper by Korstad et al. (1989) by the fact that depending on the type of algae fed, the slope of the regression line of the death rates changes from 1.1 for Tetraselmis algae to 1.9 for Nannochloris algae.

\section{Main features}

In Table A1 are summarized several former observations of rotifer mortality. All these results were published in the form of survivorship curves which give the proportion surviving as a function of age. Being steadily decreasing by their very definition all such curves have same shape which makes their interpretation and comparison difficult. That is why in Fig.A1 and Table A1 we have given several indicators which facilitate the comparison. This was done through the following operations. 
(1) Usually the survivorship curves start with a flat section during which not a single death has occurred. The width of this flat part is given in Table 1; its average is 48 hours that is to say 2 days.

(2) The next column of Table A1 gives the time it takes for one half of the population to die. Its average is 8.5 days. Note that this is probably an over-estimate mostly due to case 4. A separate paper, namely Gopakumar (2004), gives a life span estimate of 5.7 days.

(3) For each time interval $\Delta t$ between successive data points we computed the death rate: $\mu=[1 / s(t)] \Delta s / \Delta t$, where $s(t)$ represents the number of survivors at age $t$.

\section{Comments about variability}

The results given in Fig.A1 and Table A1 show a high degree of variability. How can it be explained?

In raising rotifers there are four main parameters that one can control: (i) strain identity, (ii) salinity, (iii) quantity and quality of food, (iv) temperature. In selecting the cases we tried to be as close as possible to "standard" conditions. The variability brought about by a change in food can be seen by comparing Sun (1) and Sun (2). In Johnston (2016) it was shown that death rates are markedly reduced under lower temperatures (for instance $16^{\circ} \mathrm{C}$ ) but the case that we have selected correspond to standard room temperature. The salinity level is known to have an important impact on the growth rate; for our selected cases the salinity is indicated at the end of the notes of Table 1; here too our cases correspond to fairly standard levels. In short, it seems difficult to explain the huge variability by the parameters of the experiments. The small size of the samples that were tested certainly played a role as confirmed by the large fluctuations of individual curves. However, random fluctuations cannot alone explain such huge discrepancies. The early values of curve 3 are 10 times higher than those of curve 2; similarly the early values of curve 5 are about 10 times higher than those of curve 4 .

A reasonable assumption would be to attribute such discrepancies to differences in methodology and procedure. One can mention the following problem. In principle the time axis of Fig.3 is supposed to record the post-hatching age. However, as hatching can hardly be observed individually the definition of this variable depends upon the experimental procedure. Remember that the main objective of all these papers was not to measure the death rate of rotifers in normal conditions. Such curves were merely drawn as controls for more ambitious goals, for instance to study the influence of temperature or the toxicity of flame retardants. 


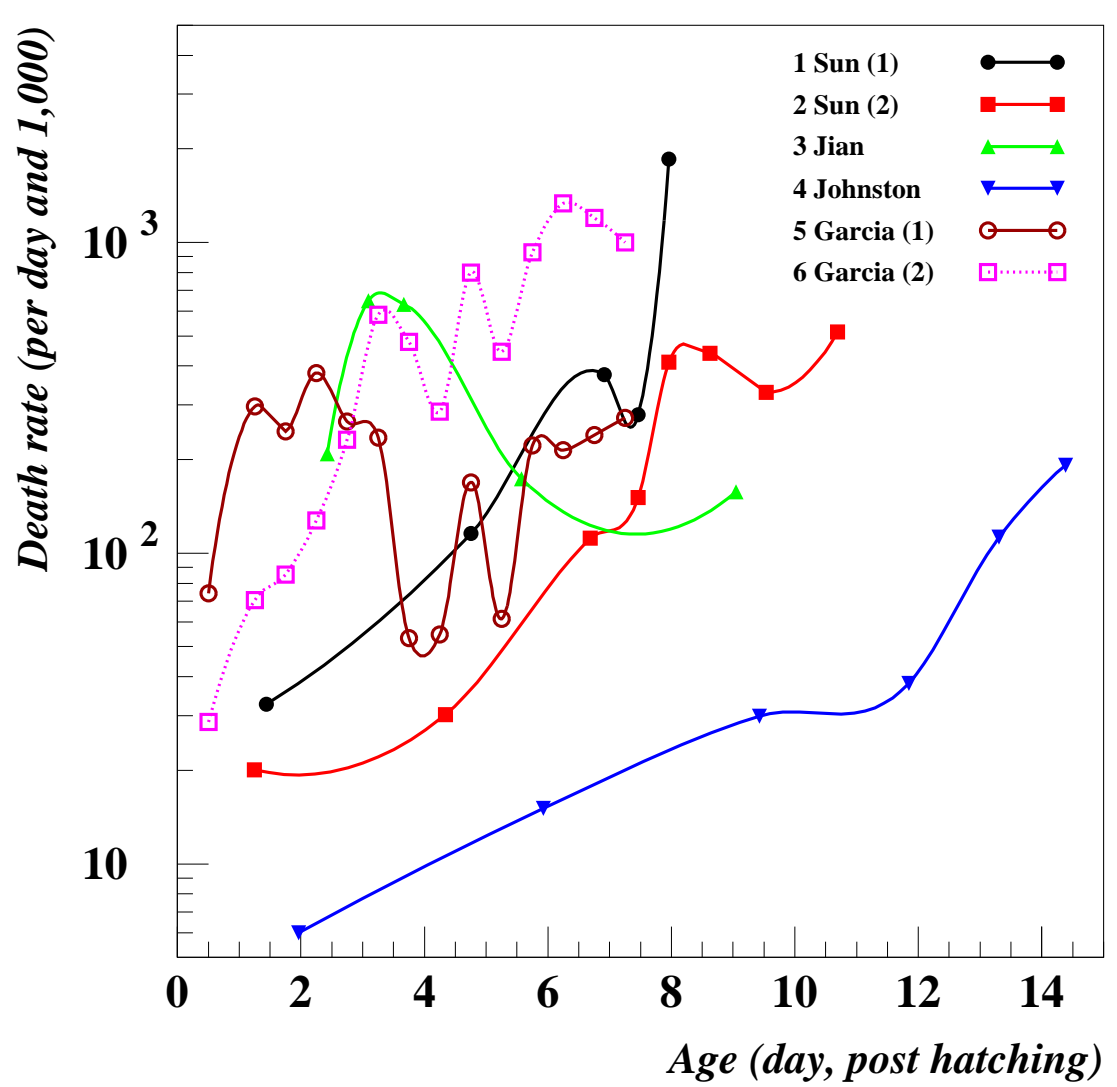

Fig. A1 Age-specific mortality rates for populations of Brachionus plicatilis. Note that the curves are different not only in shape but also in death rate levels. Case 4 was in fact based on B. manjavacas, a sister species of B. plicatilis. Sources: Sun et al. (2017); Jian et al. (2017); Johnston et al. (2016); Garcia-Roger et al. (2006)

There are two questions: (i) Is there a phase of infant mortality characterized by declining death rates. (ii) If there is one, how long does it last? Can the survivorship data of Table A1 and Fig.A1 help us to solve these questions?

The second question is easier than the first. As the sections of the curves of Fig.A1 in the moments immediately after hatching are increasing, one is led to the conclusion that if these data are correct the infant mortality is certainly shorter than 12 hours.

At first sight it may appear that the results of Table A1 give a negative answer to question (i). Indeed, the flat starting parts of the survivor curves mean that there are zero death during these intervals. Thus, it would seem, there can be no infant deaths in the hours following birth. In truth, however, a real death rate is never equal to zero. Provided that the initial sample is large enough, there will always be a few deaths. When for an initial population of 24 no deaths are observed during the first 12 hours, it simply means that the probability of death is smaller than $1 / 24$ for a $12 \mathrm{~h}$ interval, that is to say: $1 /(24 \times 12)=3.5 \times 10^{-3}$ per hour.

In addition, in the initial stage it is difficult to distinguish between eggs which do not 
Table A1: Review of survivorship experiments for rotifers (Brachionus plicatilis).

\begin{tabular}{|c|c|c|c|c|c|c|c|c|}
\hline & Reference & Case & $n$ & Iter. & $\begin{array}{l}\text { Zero neonat. } \\
\text { death rate } \\
\text { for } \ldots \\
\quad \text { (hours) }\end{array}$ & $\begin{array}{l}\text { Time } \\
\text { to } 50 \% \\
\text { survivors } \\
\text { (days) }\end{array}$ & $\begin{array}{c}\text { Average } \\
\text { death rate } \\
(1000 \times \text { day })^{-1}\end{array}$ & $\begin{array}{c}\text { Gompertz } \\
\text { exponent } \\
\beta \\
\left(\text { day }^{-1}\right)\end{array}$ \\
\hline 1 & Sun 1 & Food 1 & 24 & 3 & 60 & 7.5 & $530 \pm 660$ & $0.51 \pm 0.26$ \\
\hline 2 & Sun 2 & Food 2 & 24 & 3 & 60 & 8.7 & $250 \pm 135$ & $0.39 \pm 0.11$ \\
\hline 3 & Jian & Control & 240 & 1 & 36 & 3.0 & $364 \pm 222$ & $-0.16 \pm 0.24$ \\
\hline 4 & Johnston & $22^{\circ} \mathrm{C}$ & 120 & 1 & 72 & 15 & $65 \pm 58$ & $0.26 \pm 0.06$ \\
\hline 5 & Garcia 1 & Asexual & 72 & 1 & 12 & 3.5 & $185 \pm 51$ & $0.08 \pm 0.2$ \\
\hline \multirow[t]{2}{*}{6} & Garcia 2 & Sexual & 72 & 1 & 12 & 3.6 & $539 \pm 220$ & $0.53 \pm 0.12$ \\
\hline & Average 1-4 & & & & $48 \pm 10$ & $8.5 \pm 5$ & $279_{ \pm 80}$ & $0.21 \pm 0.12$ \\
\hline
\end{tabular}

Notes: All these references are collective papers. Please check the reference section for the names of the coauthors. "Case" means that the paper contains several survivorship observations made in different conditions of which we selected one or two, for instance with respect to food (as in 1,2), temperature (as in 4) or reproduction style (as in 5,6). $n$ denotes the size of the sample. "iter" refers to the number of iterations, i.e. (repeat) experiments. The column "Zero neonatal death rate" means that after the start of the experiment the first death was recorded at the time $t$ given in the column, in other words the survivorship curve is flat from 0 to $t$. The "average death rate" is the time-average of all recorded death rates. The last column gives the exponent $\beta$ of the Gompertz law defined by $\mu \sim \exp (\beta t)$ where $\mu$ is the death rate and $t$ the age. The average does not include 6 because it is a process of a different nature. Salinity levels were the following. 1,2,3:33g/liter (seawater); 4:unknown; 5,6: 12g/liter.

Sources: Sun et al. (2017); Jian et al. (2017); Johnston et al. (2016); Garcia-Roger et al. (2006)

hatch and neonates which die shortly after hatching. In the paper by Garcia-Roger et al. (2006, p. 259) this is expressed through the following remark.

"Two neonates died before the first observation (12h), and they were discarded

for the life table computations, since it was assumed that they were unable to complete successful emergence."

The same difficulty arises in human statistics when one needs to discriminate between stillbirths and early neonatal deaths. As infant mortality refers to live births, the second cases should be included whereas the first should not.

\section{Appendix B: Methodology of the rotifer experiment}

\section{Procedures}

The rotifers were raised in a big container of several liters ( $15 \mathrm{~g}$ of salt per liter) which was supplied with food (from a French commercial company) and bubble aeration for oxygen renewal. Ideally, from this tank one would wish to extract as many eggs as possible. There are two main obstacles. 
- The eggs are produced inside the body of the females, then at some point they are expelled but remain attached to the rear part of the body. They may hatch while still attached to the body of the female; alternatively some eggs may first get detached before hatching. Thus, in a sample of rotifers the free eggs represent only a small percentage (of the order of 10\%) of all the existing eggs. The obvious conclusion is that in order to get a large number of eggs one must detach the eggs from the body of the mother. Various means can be used. In our case we used a syringe to first take in and then expel the liquid. The jet could be directed either against the wall of the container or the surface of the liquid. Both ways were equally effective in terms of separation of the eggs; most of the time we have been using the second.

- The previous procedure produces a liquid containing a large number of free eggs, and also a substantial number of living and dead rotifers. Whereas the living rotifers are swimming at various levels, the dead ones as well as the eggs fall to the bottom. One may think that this gives a way for separating the eggs from the living but this method gives disappointing results because although swimming rotifers can indeed be found at all levels they are in particularly high concentration near the bottom. Alternatively, we used a procedure (illustrated in Fig. B1) consisting in rotating the Petri dish in a way which concentrates the swimming rotifers as well as the dead and eggs at the center. After that one needs only to wait some time until most of the swimmming rotifers have spread to the rest of the dish.

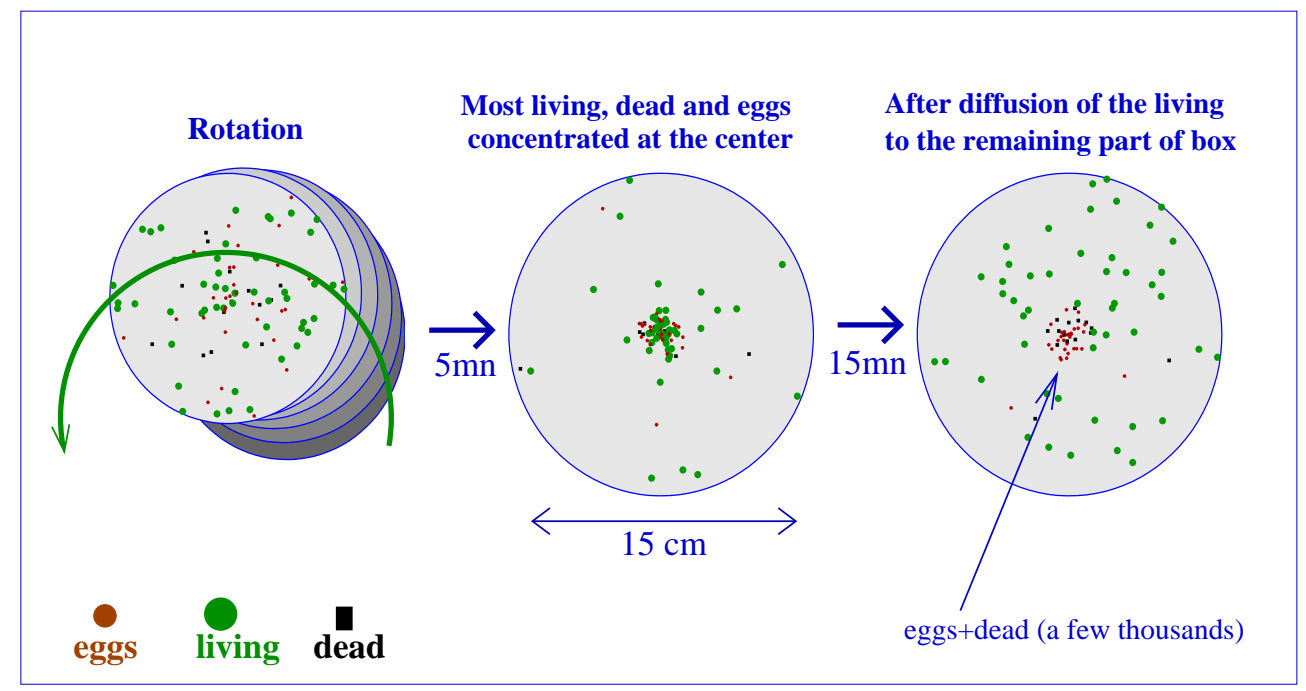

Fig. B1 Extraction of the eggs and dead rotifers. By rotating a large Petri dish $(15 \mathrm{~cm}$ or $19 \mathrm{~cm}$ in diameter so that its center follows a small circle, all living or dead rotifers and all eggs will drift to the center of the dish. In a second step the living rotifers will leave the center area and spread to the rest of the dish. In this way one can collect the eggs (and dead rotifers) with a minimum number of living rotifers.

\section{Operational definition of death}

Between normal activity and death there is a gradual transition marked by successive stages which are summarized in Table B1. This is due to the fact already mentioned 



Fig. B2a,b Two methods for measuring infant mortality rates of rotifers. In method 1 it is assumed that one can get a set of eggs which not mixed with dead rotifers. In the production conditions of our lab this condition was not fulfilled. In addition, discarding non-hatched eggs would be very time consuming which is why this method was not used. In the second method the dead rotifers and the non-hatched eggs reamain at the bottom of the eppendorf tube. One drawback of this method is that one cannot measure the number of rotifers which die immediately after hatching. For that reason, this method was used only marginally.

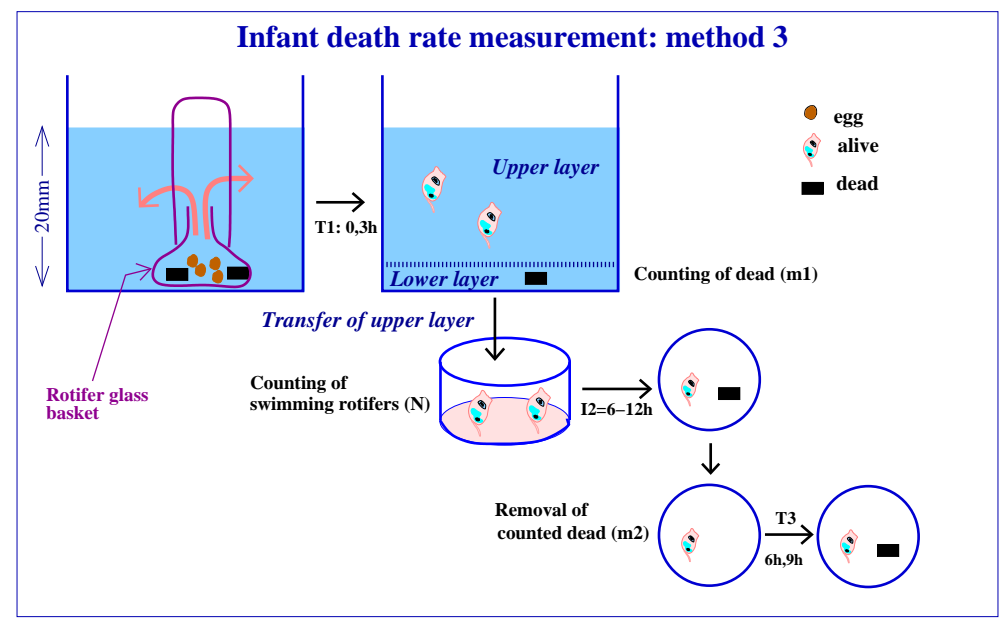

Fig. B2c A third method for measuring infant mortality rates of rotifers. It is this method which was mainly used in the present study. The dead and non-hatched eggs remain inside the basket. The rotifers which die in the first three hours, i.e. soon after being born can be counted (provided they can emerge from the basket).

previously that rotifers have few vital organs. Therefore their death is more alike the withering of a plant than the well defined death of a mammal. To ascertain that a rotifer is dead one would need to observe it for at least $5 \mathrm{mn}$ in order to make sure that there are no residual movements. While this is possible for very small samples of less than 20 individuals, it becomes unpractical for samples of several hundreds. Before we describe the solution we adopted two remarks are in order.

- Quite surprisingly this question is hardly discussed in previously published papers.

- Whatever stage is adopted as an operational definition of death one must apply it consistently in the successive phases of an experiment. Moreover one must check 
Table B1: Stages between normal activity and death

\begin{tabular}{|c|c|c|}
\hline \multicolumn{2}{|r|}{ (A) Outside aspect } & (B) Aspect inside of organism \\
\hline 1 & Fast swimming & Activity of various organs \\
\hline 2 & Slow swimming & Frequent mastax contractions \\
\hline 3 & Slow spinning & Rare mastax contractions \\
\hline 4 & Unfrequent movements & Unfrequent quivering \\
\hline 5 & Apparent steadyness & Apparent steadyness \\
\hline 6 & No movement whatsoever=death & Prickling $=$ death \\
\hline
\end{tabular}

Notes: From 1 to 5 more and more time is required to identify the relevant stages. A few seconds is enough for the identification of stage 1 whereas for stage 4 it may take up to 5 minutes. Prickling aspect may be due to the activity of bacteria which feed on the body of the dead rotifer.

that the rotifers counted as dead under the chosen criterion are indeed in a pre-death stage. This is easy actually because as all those rotifers are transferred from the main sample to a cemetery container one needs only, at the end of the experiment, to verify that the cemetery container contains no (or almost no) swimming rotifers.

The main criterion that we have been using consists in being motionless with the body sticking to the bottom of the Petri dish. The "sticking" criterion ensures that being motionless has lasted for a while. Moreover, sticking can be identified easily for the following reason. The movement of sliding the Petri dish from left to right or from right to left induces a periodic movement of the water whose frequency depends upon the size of the dish. For a diameter of about $10 \mathrm{~cm}$ the period is of the order of one second. Thus, in addition to their own movement rotifers which are swimming vibrate in a left-right direction whereas those which stick to to the bottom do not vibrate which makes them easily recognisable. Once a possible dead has been identified in this way two additional tests are done.

- A shock is applied to the Petri dish which should induce a small and rapid vibration of the item under consideration. This vibration is due to the fact that the body of rotifers is fairly flexible. If there is no vibration the item is probably a scrap of rubbish. This test is particularly useful in the initial stage of the experiment; after a while one becomes used to discriminating rotifers from rubbish of similar size and shape.

- In order to see if there is activity inside the body of the rotifer one needs to increase the magnification. If there is no activity the rotifer will be counted as 1 dead, whereas if there is still low residual activity it will be counted as 0.5 dead. The operational meaning of this convention is that one half of the supposedly dead rotifers put in the "cemetery" may turn alive again.

\section{Appendix C. Correct estimate of the initial death rate}


In the experiment described in Fig.B2.c the first time interval is somewhat special. In subsequent time intervals there is a pure death process. In contrast, during the first time interval there is a mixed birth and death process. The birth process consists in the fact that rotifers hatch and emerge from the basket. During that same time interval a small number of rotifers die. This raises the question of how to estimate the death rate. This question is of particular interest because our main interest is what happens in the early moments after birth.

In a "normal" time interval that is to say one without births, the death rate is defined in the standard way by: $\mu=\left(1 / n_{0}\right)[m(t) / t]$ where $n_{0}$ is the number of rotifers exposed to the risk, $t$ is the length of the first time interval and $m(t)$ is the number of rotifers which die in $(0, t)$. In addition, we denote by $n(t)$ the number of rotifers which emerge from the basket in the the course of time. For the first time interval $(0, t)$ the question is: what should we take for $n_{0}$ ? Clearly it would not be correct to take $n_{0}=n(t)$ for these $n(t)$ rotifers were all present only at time $t$; in fact they were exposed to the risk only during the fraction of time between their birth and time $t$. If $n_{0}=n(t)$ is not correct, should one take $n_{0}=n(t) / 2$ ? One needs to give the matter a closer look before one can answer with certainty.

The following simple model will solve the question. We will see that $n_{0}=n(t) / 2$ is indeed acceptable but only if we make some additional assumptions.

We denote by $p d t$ the probability of the emergence of a rotifer of the basket in time $d t$ and by $q d t$ the probability for an emerged rotifer to die. Thus, according to the rules of Bernoulli trials, the change in time $d t$ of the average number of dead rotifers, $m(t)$, will be given by: $d m=n(t) q d t$. This relation means that $q$ is the death rate (usually denoted by $\mu$ ).

Thus, the number $n(t)$ of living rotifers will be described by the differential equation:

$$
d n=p d t-d m \Rightarrow \frac{d n}{d t}=p-q n \Rightarrow n(t)=\frac{p}{q}[1-\exp (-q t)]
$$

Actually, we are more interested in $m(t)$ than in $n(t)$ :

$$
\frac{d m}{d t}=\frac{p}{q}[1-\exp (-q t)] q \Rightarrow m(t)=p t-\frac{p}{q}[1-\exp (-q t)]
$$

Now we assume that $q$ is small with respect to 1 and $t$ not too large:

$$
q t \ll 1 \Rightarrow n(t) \sim \frac{p}{q}[1-(1-q t)]=p t
$$

For $m(t)$ development to first order gives 0 but to second order one obtains:

$$
m(t) \sim p q \frac{t^{2}}{2}
$$


Our estimation problem now consists, from the knowledge of: $t, m(t), n(t)$, to extract $p$ and $q$. From equations (C3) and (C4) one gets:

$$
p=n(t) / t, \quad q=\frac{m(t)}{[n(t) / 2] t}
$$

In other words, our initial guess $n_{0}=n(t) / 2$ was correct, but only in the approximation $q t \ll 1$ corresponding to a very slow death process. Otherwise, to get $p$ and $q$ the equations ( $\mathrm{C} 1),(\mathrm{C} 2)$ would have to be solved numerically.

\section{Acknowledgments}

First of all, we wish to thank Ms. Florie Lopis who deftly made small glass "baskets" in which the eggs of the rotifers could hatch and produce swimming neonates. These devices played a crucial role in the first step of each trial.

One of the co-authors (B.R.) would like to express his gratitude to the following colleagues who welcomed him in their laboratories and provided advice and guidance for setting up experiments which eventually led to the rotifer experiment described in this paper:

Fei Dou (Beijing Normal University), Patrick Dumont (Greenhouse of the "Institut de Biologie de Paris Seine (Jussieu)", Michel Gho (University Pierre and Marie Curie), Nobuhiko Suematsu (Meiji University), Kun Wang (Beijing Normal University), Claude Yéprémian (Muséum d'Histoire Naturelle, Paris).

\section{References}

Berrut (S.), Pouillard (V.), Richmond (P.), Roehner (B.M.) 2016: Deciphering infant mortality. Physica A 463,400-426.

Bois (A.), Garcia-Roger (E.M.), Hong (E.), Hutzler (S.), Ali Irannezhad (A.), Mannioui (A.), Richmond (P.), Roehner (B.M.), Tronche (S.) 2019a: Congenital anomalies from a physics perspective. The key role of "manufacturing" volatility. Preprint (April 2019).

Bois (A.), Garcia-Roger (E.M.), Hong (E.), Hutzler (S.), Ali Irannezhad (A.), Mannioui (A.), Richmond (P.), Roehner (B.M.), Tronche (S.) 2019b: Physical models of infant mortality. Implications for biological systems. Preprint (June 2019).

Carrillo (A.), McHenry (M.J.) 2015: Zebrafish learn to forage in the dark. Integrative and Comparative Biology (conference) 219,4,E26.

Declerck (S.A.), Papakostas (S.) 2017: Monogonont rotifers as model systems for the study of micro-evolutionary adaptation and its eco-evolutionary implications. Hydrobiologia 796,1,131-144. 
Depree (J.A.), Geoffroy (P.S.) 2001: Physical and flavor stability of mayonnaise. Trends in Food Science and Technology 12,5,157-163.

Garcia-Roger (E.M.), Martínez (A.), Serra (M.) 2006: Starvation tolerance of rotifers produced from parthenogenetic eggs and from diapausing eggs: a life table approach. Journal of Plankton Research 28,3,257-265.

Gerhard (G.S.), Kauffman (E.J.), Wang (X.), Stewart (R.), Moore (J.L.), Kasales (C.), Demidenko (E.), Cheng (K.C.) 2002: Life spans and senescent phenotypes on two strains of Zebrafish (Danio rerio). Experimental Gerontology 37,10551068.

Gisbert (E.), P. Williot (P.), Castell-Orvay (F.) 2000: Influence of egg size on growth and survival of early stages of Siberian sturgeon (Acipenser baeri) under small scale hatchery conditions. Aquaculture 183,1,83-94.

Gopakumar (G.), Jayaprakas (V.) 2004: Life table parameters of Brachionus plicatilis and $B$. rotundiformis in relation to salinity and temperature. Journal of the Marine Biological Association of India 46,1,21-31.

Grove (R.D.), Hetzel (A.M.) 1968: Vital statistics rates in the United States, 19401960. United States Printing Office, Washington, DC.

Hosoya (S.), Lu (Z.), Ozaki (Y.), Takeuchi (M.), Sato(T.) 2007: Cytological analysis of the mother cell death process during sporulation in Bacillus subtilis. Journal of Bacteriology 189,6,2561-2565.

Jian (X.), Tang (X.), Xu (N.), Sha (J.) YouWang (Y.) 2017: Responses of the rotifer Brachionus plicatilis to flame retardant (BDE-47) stress. Marine Pollution Bulletin 116,1-2,298-306.

Johnston (R.K.), Snell (T.W.) 2016: Moderately lower temperatures greatly extend the lifespan of Brachionus manjavacas (Rotifera): Thermodynamics or gene regulation? Experimental Gerontology 78,12-22.

Jones et al. (14 co-authors) 2014: Diversity of ageing across the tree of life. Nature 505,169-173.

Joshi (P.S.) 1988a: Influence of salinity on population growth of a rotifer, Brachionus plicatilis. Journal of the Indian Fisheries Association 18,75-81.

Joshi (P.S.) 1988b: Mass culture of Brachionus plicatilis. Master of Science Dissertation, University of Bombay (84 p.) [available on line]

Kioumourtzoglou (M.-A.), Coull (B.A.), O'Reilly (E.J.), Ascherio (A.), Weisskopf (M.G.) 2018: Association of exposure to diethylstilbestrol [DES] during pregnancy with multigenerational neurodevelopmental deficits. Journal of the American Medical Association (JAMA), Pediatrics. 172,7,670-677. 
Kohler (I.V.), Preston (S.H.), Lackey (L.B.) 2006: Comparative mortality levels among selected species of captive animals. Demographic Research volume 15, article 14, p.413-434.

Korstad (J.), Olsen (Y.), Vadstein (O.) 1989: Life history characteristics of Brachionus plicatilis (rotifera) fed different algae. Hydrobiologia 186/187,43-50.

Linder (F.E.), Grove (R.D.) 1947: Vital statistics rates in the United States, 19001940. United States Printing Office, Washington, DC, 1947.

Miyo (T.), Charlesworth (B.) 2004: Age-specific mortality rates of reproducing and non-reproducing males of Drosophila melanogaster. Proceedings of the Royal Society B 271,2517-2522.

Ødegaard (F.) 2000: How many species of arthropods? Erwin's estimate revised. Biological Journal of the Linnean Society 71.583-597.

Pearl (R.), Miner (J.R.) 1935: Experimental Studies on the duration of life. XIV. The comparative mortality of certain lower organisms. The Quarterly Review of Biology 10,1,60-79.

Pearl (R.), Park (T.), Miner (J.R.) 1941: Experimental studies on the duration of life. XVI Life tables for the flour beetle Tribolium confusum Duval. The American Naturalist 75,756,5-19.

Pouillard (V.) 2015: En captivité. Vies animales et politiques humaines dans les jardins zoologiques du XIXe siècle à nos jours: ménagerie du Jardin des Plantes, zoos de Londres et Anvers (Ph.D. thesis), Université Libre de Bruxelles and Université de Lyon, [In captivity. Zoo management and animal lives in the zoological gardens of Paris, London and Antwerp from the 19th century to 2014].

Rombough (P.J.) 1998: Partitioning of oxygen uptake between the gills and skin in fish larvae: a novel method for estimating cutaneous oxygen uptake. Journal of Experimental Biology 201,11,1763-1769.

Sahin (T.) 2001: Larval rearing of the Black Sea Turbot, Scophthalmus maximus (Linnaeus 1758), under laboratory conditions. Turkish Journal of Zoology 25,447-452.

Snell (T.W.), Janssen (C.) 1995: Rotifers in ecotoxicology: a review. Hydrobiologia 313,231-247.

Strehler (B.L.) 1967: Mortality Trends and Projections. Society of Actuaries (October)

Sun (Y.), Hou (X.), Xue (X.), Zhang (L.), Zhu (X.), Huang (Y.), Chen (Y.), Yang (Z.) 2017: Trade-off between reproduction and lifespan of the rotifer Brachionus plicatilis under different food conditions. Scientific Reports 7,1.

Tarazona (E.), Garcia-Roger (E.M.), Carmona (M.J.) 2017: Experimental evolution 
of bet hedging in rotifer diapause traits as a response to environmental unpredictability. Oikos 126,1162-1172.

Wallden (M.), Fange (D.), Lundius (E.G.), Baltekin (Ö.), Elf (J.) 2016: The synchronization of replication and division cycles in individual E. coli cells. Cell 166,729-739. 